Die Argumentation mit dem Matriarchat im Spannungsfeld zwischen Aufklärung und Esoterik

\author{
Fehlmann, Meret
}

Posted at the Zurich Open Repository and Archive, University of Zurich

ZORA URL: https://doi.org/10.5167/uzh-80563

Book Section

Published Version

Originally published at:

Fehlmann, Meret (2013). Die Argumentation mit dem Matriarchat im Spannungsfeld zwischen Aufklärung und Esoterik. In: Neugebauer-Wölk, Monika; Geffarth, Renko; Meumann, Markus. Aufklärung und Esoterik : Wege in die Moderne. Berlin: De Gruyter, 785-805. 


\section{Die Argumentation mit dem Matriarchat im Spannungsfeld zwischen Aufklärung und Esoterik}

Im Internet eröffnet sich eine ,matriarchale Landschaft‘. Mit seinen Möglichkeiten, Informationen zu vermitteln, Netzwerke aufzubauen und gleichgesinnte Menschen zu erreichen, kann das Internet zu einer zweiten Heimat für neue religiöse Bewegungen werden, denen es als interaktives Gefäß für religiöse Praxis und Ritualausübung dient. ${ }^{1}$ Eine dieser neuen religiösen Bewegungen stellt der spirituelle Feminismus dar, in dessen Weltanschauung das Matriarchat eine wichtige Rolle spielt. Auf der Seite Gynozentrisches Knistern - Kessel der Inspiration für Hexenweiber ${ }^{2}$ begegnen uns zentrale Elemente der gegenwärtigen Argumentation mit dem Matriarchat:

Unsere VorfahrInnen kannten sie gut, die allgegenwärtige weibliche Kraft. Auf dieser Kraft beruhte die Ordnung - der Rhythmus von Leben und Vergehen, von Ebbe und Flut, vom Aufgang und vom Untergang der Sonne. [...] Und weil die frühen Menschen die Analogien zwischen den Naturerscheinungen und der Frau erkannten, wurde die Frau zum Symbol kosmischer, weiblicher Schöpfungskraft. Die Große Mutter oder Große Göttin war der Inbegriff aller Lebensordnung, die schöpferische Urkraft, die matriarchale Weisheit. ${ }^{3}$

Dieser Textausschnitt stammt von Hannelore Vonier $(* 1951)$, die mehrere Homepages zum Thema unterhält. ${ }^{4}$ Der Text weist eine für den spirituellen Feminismus charakteristische Engführung von Weiblichkeit und Natur auf, die sich im gleichen Rhythmus befinden sollen. Aus der postulierten Naturnähe der Frau resultiert die ihr zugeschriebene Heiligkeit. Dabei handelt es sich um ein typisches Argumentationsmuster innerhalb des Matriarchatsdiskurses. Dieses Beispiel zeigt, dass sich die matriarchale Argumentation stark auf die traditionellen Geschlechtscharaktere stützt, wo Weiblichkeit mit Körperlichkeit und Emotionen in Verbindung gebracht wird. In der westlichen Welt ist bis heute eine Dichotomie von als weiblich und als männlich verstandenen Eigenschaften auszumachen, diese Zuschreibungen bezeichnet man als Geschlechtscharaktere. Sie entstanden im 18. Jahrhundert, verfestigten sich im 19. Jahrhundert und konnten sich bis heute halten, obwohl sie im

1 Douglas E. Cowan: Cyberhenge. Modern Pagans on the Internet. New York 2005, S. 18f., S. 113-116.

2 URL: http://www.spiritvoices.de/knistern/hanne.html [19.02.2010].

3 Ebd.

4 In deutscher Sprache die beiden Domains URL: http://www.matriarchat.info [13.11.2009] und URL: http://www.matriarchat.net [13.11.2009]. Auf Englisch führt sie URL: http://pro matriarchy.net [13.11.2009]. Alle drei Websites sind auf ihren Namen, also Hannelore Vonier, eingetragen. Matriarchat.net und promatriachy.net sind seit 2002 auf ihren Namen eingetragen, matriarchat.info erst seit dem Jahr 2005. Überprüft mit URL: http://domains.whois.com/ domain.php?action=whois [13.11.2009]. 
Laufe des 20. Jahrhunderts zunehmend kritisiert und hinterfragt wurden..$^{5}$ Innerhalb des Matriarchatsdiskurses ist eine Umkehrung der traditionellen Wertung festzustellen: Alles Weibliche ist positiv konnotiert, das Männliche umgekehrt wird als negativ gebrandmarkt.

Im spirituellen Feminismus - einem separatistischen, auf Frauen und auf religiöse Aspekte fokussierenden Zweig der „zweiten Frauenbewegung“ in den 1960er und 1970er Jahren ${ }^{6}$ - sowie anderen esoterischen Kreisen ist der Glaube an die Existenz eines vorchristlichen Matriarchats weit verbreitet. Gemäß dieser Vorstellung soll das Matriarchat in früheren Zeiten weltweit dominierend gewesen sein. Es habe sich durch Gewaltfreiheit und Klassenlosigkeit ausgezeichnet. Ein weiteres zentrales Merkmal bildet der Kult einer Großen Göttin - verstanden als Herrin über den ewigen Kreislauf von Leben und Tod. ${ }^{7}$ Die religiöse Vormacht des weiblichen Geschlechts habe ihre Entsprechung im sozialen und politischen Bereich gehabt. Diese Gesellschaftsordnung soll in (proto-)historischen Zeiten durch das Patriarchat abgelöst worden sein. Eine solche Evozierung des Matriarchats verrät mit Zuschreibungen wie Klassenlosigkeit, Kult einer Göttin, Vormacht des Weiblichen und Verortung in der Vergangenheit die Abstammung aus verschiedensten Bereichen, darunter zahlreichen Wissenschaftszweigen und sozialen Bewegungen.

Und noch ein weiteres Charakteristikum der Argumentation mit dem Matriarchat manifestiert sich in Gynozentrisches Knistern - Kessel der Inspiration für Hexenweiber: „Im matriarchalen Bewusstsein bewegt sich die Welt in Zyklen. Alles Vergangene kehrt wieder! Und unsere Reise zu den Ursprüngen ist zugleich der Weg in unsere Zukunft." ${ }^{\text {"8 }}$ Der Bezug auf das Matriarchat spricht die Hoffnung auf die Rückkehr dieser Gesellschaftsordnung aus. Ein Rückbezug auf das als besser empfundene Matriarchat ist typisch innerhalb der sozialen Bewegungen, wie sie beispielsweise der spirituelle Feminismus darstellt. So galt und gilt ihnen das

5 Cornelia Klingler: 1800 - Eine Epochenschwelle im Geschlechterverhältnis? In: Revolution und Emanzipation. Geschlechterordnung in Europa um 1800. Hg. v. Katharina Rennhak u. Virginia Richter. Köln 2004 (Literatur-Kultur-Geschlecht. Große Reihe 31), S. 17-32, hier S. 19f.

6 Die neue oder zweite Frauenbewegung entstand im Westen in den späten 1960er und den 1970er Jahren. Ihr ging es darum, die traditionellen Geschlechterrollen aufzubrechen und aufzuheben, um so eine Emanzipation der Menschheit insgesamt zu erreichen. Im Laufe der 1970er Jahre teilte sich die zweite Frauenbewegung in verschiedene Zweige - auch mit unterschiedlichen Zielen und Bestrebungen - auf. Einer dieser Zweige ist der spirituelle Feminismus. Regina Dackweiler: Ausgegrenzt und eingemeindet. Die neue Frauenbewegung im Blick der Sozialwissenschaften. Diss. Frankfurt a.M. 1995, hier S. 7f., 120-123 und Kristina Schulz: Frauen in Bewegung. Mit der neuen Linken über die Linke(n) hinaus. In: 1968. Handbuch zur Kultur- und Mediengeschichte der Studentenbewegung. Hg. v. Martin Klimke u. Joachim Scharloth. Stuttgart 2007, S. 247-257, hier S. 247f., $253 \mathrm{f}$.

7 Hans G. Kippenberg, Kocku von Stuckrad: Geschlechterperspektiven: Auf der Suche nach der Muttergottheit. In: Dies.: Einführung in die Religionswissenschaft. Gegenstände und Begriffe. München 2003, S. 81-93, hier S. 83-85.

Siehe Anm. 2. 
Matriarchat als Orientierung für die Zukunft. Mit dem Matriarchat waren und sind Heilsversprechen für die Menschheit verbunden.

Im Folgenden soll die Argumentation mit dem Matriarchat näher untersucht werden. Da das Matriarchat momentan eine feste Heimstatt im spirituellen Feminismus hat, dem esoterische Züge nicht abzusprechen sind, wird zuerst die Affinität der Argumentation mit dem Matriarchat zu esoterischen Denkformen behandelt. Da die Argumentation mit dem Matriarchat auf eine über 150 Jahre alte Geschichte zurückblicken kann, die immer wieder zu Ergänzungen und Erweiterungen ihrer Inhalte geführt hat, fasst man sie am besten als Konstrukt auf. Sodann folgt ein Überblick über die Anfänge des Matriarchats, die einerseits bei wissenschaftlichen Schriften, andererseits bei sozialen Bewegungen liegen. $\mathrm{Zu}$ einer Begeisterung für das Matriarchat kam es in der „ersten Frauenbewegung“, der angelsächsischen Frauenbewegung des ausgehenden 19. Jahrhunderts, ${ }^{9}$ die die Argumentation mit dem Matriarchat für ihren Kampf für Frauenrechte entdeckte und teilweise bereits für eine Aufladung des Matriarchats mit esoterischen Inhalten sorgte. Da die Vorstellung vom Matriarchat sich kontinuierlich weiterentwickelt hat, wurden auch problematische Argumentationsweisen, eurozentristische, rassistische oder gar antisemitische, aufgenommen.

\section{Matriarchat und Esoterik}

In den 1990er Jahren schlug Antoine Faivre vor, unter Esoterik eine spezifische Denkform zu verstehen. Faivre entwickelte sechs seiner Meinung nach charakteristische Eigenschaften oder Merkmale dieser Denkform. ${ }^{10}$ Innerhalb der aktuellen Matriarchatsargumentation tauchen einige dieser Elemente mit großer RegelmäBigkeit auf, vor allem das Denken in Entsprechungen, das Verständnis der Erde als lebender Organismus und die Transmission.

9 Die erste Frauenbewegung im 19. Jahrhundert kämpfte vor allem gegen rechtliche und soziale Einschränkungen. Frauen hatten keine politischen Rechte, ihre Bildungsmöglichkeiten waren stark eingeschränkt. In der Ehe war die Frau dem Mann zu Gehorsam verpflichtet, der Mann verfügte über das Verwaltungs- und Nutzungsrecht ihres Vermögens sowie über die elterliche Gewalt. Uneheliche Kinder galten in den europäischen Ländern per Gesetz als nicht mit dem Vater verwandt, sie waren nicht erbberechtigt, die Mutter hatte für sie aufzukommen. Zeitlich lässt sich der Beginn der ersten Frauenbewegung nicht klar festlegen, denn die theoretische Beschäftigung mit der Rolle und Stellung der Frau in der Gesellschaft geht den konkreten Forderungen und der Bildung von Organisationen und Vereinen voraus (Theresa Wobbe: Gleichheit und Differenz. Politische Strategien von Frauenrechtlerinnen um die Jahrhundertwende. Frankfurt a.M. 1989 [Campus Forschung 620], S. 46f.; Ann Taylor Allen: Feminism and Motherhood in Western Europe. 1890-1970. The Maternal Dilemma. New York 2005, S. 42; Elaine Showalter: Sexual Anarchy. Gender and Culture at the Fin de Siècle. Hammondsworth 1990, S. 19f.).

10 Siehe Antoine Faivre: Esoterik im Überblick. Freiburg i.Br. 2001 (Herder Spektrum 4961), S. 24-32. Die sechs Elemente sind: Denken in Entsprechungen/lebende Natur/Imagination und Mediation/die Erfahrung der Transmutation/Konkordanzbildung/Transmission. 
Weit verbreitet unter den Exponentinnen und Exponenten des Matriarchats ist ein Denken in Entsprechungen, wonach alle Teile der realen und der unsichtbaren Welt miteinander in Verbindung stehen. Deutlich bringt Christa Mulack (*1943), eine deutsche Theologin und Autorin, in Die Wurzeln weiblicher Macht (1996) die Überzeugung zum Ausdruck, dass die matriarchale Kultur um diese Verbindung wusste:

Ein Blick auf das Weltbild dieser Menschen zeigt, dass sie sich an der einfachen Formel orientierten: Wie im Kleinen, so im Großen, wie unten, so oben, wie auf Erden, also auch am Himmel. Nach dieser Regel wurde in Anlehnung an den Körperraum der Frau auch der kosmische Raum als weiblich aufgefasst. ${ }^{11}$

Ebenfalls ein wiederkehrendes Motiv ist die Überzeugung der durchgängigen Beseeltheit der Natur. Diese wird gekoppelt mit dem vorgeblichen Wissen darum, dass die Menschen in den alten Matriarchaten im Einklang mit der Erde und der Natur und ihren Rhythmen lebten und dass die Frauen durch ihre Menstruation in den Zyklus der Natur eingebunden waren. ${ }^{12}$ Es kann aber auch die Vorstellung auftreten, dass das Patriarchat nur eine vorübergehende Epoche sei, bevor sich das Matriarchat wieder ausbreiten werde. Sehr deutlich findet sich diese Haltung in Elizabeth Gould Davis' (1910-1974) Am Anfang war die Frau (1977). ${ }^{13}$ Wegen seines Lobgesanges auf die Größe der alten Matriarchate und die Bedeutung der Frau als erstem Geschlecht gilt dieses Buch häufig als Ausgangspunkt des spirituellen Feminismus. Davis zufolge sind Frauen die Initiatorinnen sämtlicher kultureller Errungenschaften, und die patriarchale Ära stelle nur eine vorübergehende, von der Sternenkonstellation verursachte Epoche dar, auf die bessere Zeiten folgen werden: „Heute wie damals sind die Frauen die Vorkämpferinnen der heraufdämmernden neuen Zivilisation; und die Frauen sind es, auf die wir bauen bei der Erlösung in den heilenden und erneuernden Wassern des Wassermanns."14

Der Bezug auf das astrologische Wassermannzeitalter verweist auf einen esoterischen Kontext, denn die moderne Theosophie und weitere esoterische Weltanschauungen verknüpften den Anbruch dieses Zeitalters in der Mitte des 20. Jahrhunderts mit der Hoffnung auf einen tief greifenden sozialen Transformationsprozess, der viele positive Veränderungen mit sich bringen werde. Diese Idee griffen die kalifornischen Protestbewegungen der 1960er und 1970er Jahre auf und verbreiteten sie. ${ }^{15}$ Bei Gould Davis bezog sich dieser Transformationsprozess der

11 Christa Mulack: Die Wurzeln weiblicher Macht. München 1996, S. 81.

12 Cynthia Eller: Living in the Lap of the Goddess. The Feminist Spirituality Movement in America. New York 1993, S. 168f; dies.: The Myth of Matriarchal Prehistory. Why an Invented Past Won't Give Women a Future. Boston 2000, S. 46f., 56f.

13 Das amerikanische Original erschien 1971 u.d.T. The first sex.

14 Elizabeth Gould Davis: Am Anfang war die Frau. München 1977, S. 349.

15 Christoph Bochinger: ,New Age' und moderne Religion. Religionswissenschaftliche Analysen. Diss. München. Gütersloh 1994, S. 308f., 333-338 und Kocku von Stuckrad: Was ist Esoterik? Kleine Geschichte des geheimen Wissens. München 2004, S. $228 \mathrm{f}$. 
Menschheit auf die baldige Rückkehr zum Matriarchat und damit die Genesung von Natur und Menschen; in ihrem Verständnis oblag den Frauen die Hauptaufgabe bei der Errichtung dieses besseren Zeitalters. Den Frauen wurde die Fähigkeit zur Reparatur der Zivilisationsschäden zugeschrieben, als weiblich verstandene Eigenschaften wurden zur Heilung benötigt. Beides lässt sich bis zu den Anfängen der Matriarchatsbezüge zurückverfolgen.

Esoterik an sich ist ein unscharfer Begriff, der verschiedenste Inhalte und Lehren umfasst. Von früheren Definitionsversuchen, die unter Esoterik eine Lehre verstanden, die einzig den Eingeweihten zugänglich ist, hat man sich mittlerweile als zu eng verabschiedet. Dennoch bezieht sich die Argumentation mit dem Matriarchat gerne auf die Vorstellung, dass das alte matriarchale Wissen im Verborgenen überdauert habe und nur darauf warte, von den richtig vorbereiteten, das heißt von den aus patriarchalen Zwängen befreiten Menschen verstanden und erneut erweckt zu werden. Was in anderen esoterischen Schulen oft die geheimen Bücher sind, wird in der Argumentation mit dem Matriarchat zum heimlich bewahrten Wissen in Märchen und anderen mündlichen Überlieferungen. Dies verweist wiederum auf die oftmals anzutreffende Überzeugung, dass sämtliches esoterisches Wissen seinen Ursprung in einer fernen Vergangenheit habe. ${ }^{16}$ Diese Denkfigur, dass altes, vorzeitliches Wissen in den mündlich tradierten Erzählstoffen seiner Entschlüsselung harre, erinnert stark an die Überzeugungen, die im 19. Jahrhundert die sogenannte Mythologische Schule hegte. Diese entwickelte sich aus der deutschen Philologie der Romantik und ging davon aus, dass sich in der mündlichen Überlieferung noch älteres, heidnisches, insbesondere germanisches Gedankengut auffinden lasse und so Zeugnis von den heidnischen Ursprüngen ablege. Ihr Streben richtete sich darauf, diese Überlieferung zu sichern, um aus ihr heraus die Urform zu suchen und zu rekonstruieren. ${ }^{17}$ Die Mythologische Schule sollte mit diesem Ansatz einen nicht zu unterschätzenden Einfluss auf die Matriarchatsdebatte ausüben. Oft genug ist das Heilsversprechen des Matriarchats an die Überzeugung gekoppelt, dass das matriarchale Geheimwissen im Verborgenen schlummere und nur darauf warte, wiederentdeckt und entschlüsselt zu werden, um seine heilsame Wirkung zu entfalten. Bis heute gilt Matriarchatsverfechterinnen und verfechtern das angenommene Weiterleben heidnischer, matriarchaler Überlieferung in Sagen, Märchen und anderen literarischen Gattungen als wichtiges methodisches Rüstzeug, auf das sie ihre Überlegungen und Arbeiten stützen. Viele

16 Vgl. Pierre A. Riffard: The Esoteric Method. In: Antoine Faivre, Wouter J. Hanegraaff (Hg.): Western Esotericism and the Science of Religion. Leuven 1998, S. 63-74, hier S. 65, 67; Antoine Faivre: Questions of Terminology Proper to the Study of Exoteric Currents in Modern and Contemporary Europe. In: Ebd., S. 1-10, hier S. 1-7.

17 Siehe Kathrin Pöge-Alder: Mythologische Schule. In: Enzyklopädie des Märchens. Handwörterbuch zur historischen und vergleichenden Erzählforschung. [Bisher erschienen] $13 \mathrm{Bde} . \mathrm{Hg}$. v. Rolf Wilhelm Brednich u.a. Bd. 9: Magica-Literatur - Nezāmi. Berlin, New York 1999, S. 1086-1092, hier S. 1086f. 
Werke, die vom Geist des spirituellen Feminismus geprägt sind, postulieren, dass Märchen und Sagen Reste der matriarchalen Weltsicht und Weisheit enthalten. Ihre Deutungsweise geht weiter, indem sie diesen Resten bis heute ein subversives, revolutionäres Potential zuschreiben, das es wiederzuerwecken gelte. So lässt

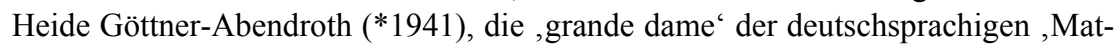
riarchatsforschung', in Das Matriarchat (1988) verlauten, dass sich Überreste der ,matriarchalen Kulturepoche“ nicht nur in der Vergangenheit finden lassen, sondern in geographischen Randzonen und in Subkulturen bis in die Gegenwart hinein lebendig blieben, denn da sei der Anpassungsdruck durch das Patriarchat weniger stark ausgefallen. ${ }^{18}$ Als Trägerschaft macht sie unter anderem die Bauern aus. Damit nimmt sie ein weiteres zentrales Element der Mythologischen Schule auf, die überzeugt war, dass die Bauern als statischer Stand von Urbanisierung und Industrialisierung nicht betroffen waren und so die alten Überlieferungen in besonders reiner Form bewahrten. Dieser Mechanismus zeigt auch, wie sich veraltete, innerhalb der Wissenschaft verabschiedete Vorstellungen weiter verbreiten können. Göttner-Abendroth versteht die Zaubermärchen und die höfischen Epen des Mittelalters als Hort dieser versteckten und verdrängten Überlieferung. ${ }^{19}$

Seit einiger Zeit erfreuen sich in der Belletristik Romane, die das Zusammentreffen und den anschließenden Kampf der frauenzentrierten Gesellschaft mit dem Patriarchat schildern, großer Beliebtheit. Dieses Erzählmuster ist insbesondere seit den 1980er Jahren durch das Erscheinen von Marion Zimmer Bradleys (19301999) Neubearbeitung der Artussage Die Nebel von Avalon ${ }^{20}$ populär geworden. Das Buch deutet die Artussage als Zeugnis des Kampfes der matriarchalen Naturreligion mit dem patriarchalen Christentum, und die Wiedergabe der Ereignisse erfolgt aus weiblicher Perspektive. Dem Buch kommt zudem eine Rolle zu bei der Verbreitung und Popularisierung eines matriarchalen Weltbildes, und es diente als Vorbild für weitere Bearbeitungen des Artusstoffes mit ähnlicher Stoßrichtung. ${ }^{21}$ Wichtig ist festzuhalten, dass in den 1980er Jahren Romane, die diesem Handlungsschema folgten, nicht mehr sonderlich neu waren. Bereits in den 1940er Jahren zeichnete der Dichter Robert Graves (1895-1985), dem eine einflussreiche Rolle bei der Ausgestaltung des Bildes der dreifaltigen Göttin um die Mitte des letzten Jahrhunderts zukam, in Romanen den Zusammenstoß der matriarchalen und patriarchalen Welt nach. Ebenso schuf der US-amerikanische Schriftsteller Vardis Fisher (1895-1968) in den 1940er Jahren mit seinem vierbändigen Werk The testament of man einen Zyklus, der die Entwicklung der Welt und Gesellschaft seit den Anfängen bis etwa zum Einsetzen der im Alten Testament geschilderten

18 Heide Göttner-Abendroth: Das Matriarchat. 3. Aufl. Stuttgart 1995, S. 116.

19 Ebd., S. 116-118, 166.

20 Das amerikanische Original erschien 1982 unter dem Titel The mists of Avalon.

21 Eller: Living (wie Anm. 12), S. 34; Julia Iwersen: Art. Artusroman. In: Lexikon der Esoterik. Hg. v. ders. Düsseldorf 2001, S. 34; Meret Fehlmann: Frauengestalten in populären Artusromanen der Gegenwart. In: Fabula 46, 3/4 (2005), S. 217-240. 
Ereignisse darstellt. Bei einem solchen Unterfangen der Wiedergabe der Geschichte der menschlichen Vorzeit und der alten Kulturen durfte das Matriarchat nicht fehlen: Der vierte Band Intimations of Eve (1946) handelt vom Untergang des Matriarchats. $^{22}$

\section{Anfänge des Konzepts vom Matriarchat}

Dank der Schrift Das Mutterrecht. Eine Untersuchung über die Gynaikokratie der alten Welt nach ihrer religiösen und rechtlichen Natur (1861) gilt Johann Jakob Bachofen (1815-1887) als ,Vater' des Matriarchats. Bachofen entstammt einer reichen Basler Patrizierfamilie. Nach dem Studium der Jurisprudenz in Berlin, Göttingen und Basel wurde er 1841 Professor für Römisches Recht an der Universität Basel, legte aber bereits nach zwei Jahren seine Professur nieder. Die Erträge des Familienvermögens sicherten ein komfortables Leben, und fortan widmete er sich seinen geschichtsphilosophischen Entwürfen und Visionen. ${ }^{23}$ Bachofen leitete aus antiken Berichten über mutterrechtliche oder matriarchale Zustände bei fremden Ethnien eine Theorie über die Entwicklung der Menschheit ab und machte drei Entwicklungsstufen aus: Hetärismus, Gynaikokratie und Vaterrecht. Die ersten beiden sind seiner Auffassung nach vom stofflichen Prinzip geprägt, die letzte Stufe, diejenige des Vaterrechts, kulminiert im geistigen, durch den Mann vertretenen Prinzip. Das geistige Prinzip geriet seiner Meinung nach durch die fortschreitende Urbanisierung und Industrialisierung und die damit einhergehenden Veränderungen unter Druck. ${ }^{24}$ Auch wenn bei Bachofen das Vaterrecht oder Patriarchat den höchsten Entwicklungsstand einnimmt, so wird doch die Sehnsucht nach der Zeit des Matriarchats deutlich: „Die gynaikokratische Weltperiode ist in der Tat die Poesie der Geschichte. Sie wird dies durch die Erhabenheit, selbst durch die Schönheit, zu der sie das Weib erhebt, durch die Beförderung der Tap-

22 Nicholas Ruddick: The Fire in the Stone. Prehistoric Fiction from Darwin to Jean M. Auel. Middletown 2009, S. 73-75.

23 Andreas Cesena: Johann Jakob Bachofens Geschichtsdeutung. Eine Untersuchung ihrer geschichtsphilosophischen Voraussetzungen. Diss. Basel. Basel, Boston u. Stuttgart 1983 (Basler Beiträge zur Philosophie und ihrer Geschichte 9), S. 67; Eckhard Heftrich: Johann Jakob Bachofen und seine Bedeutung für die Literatur. In: Mythos und Mythologie in der Literatur des 19. Jahrhunderts. Hg. v. Helmut Koopmann. Frankfurt a.M. 1979 (Studien zur Philosophie und Literatur des 19. Jahrhunderts 36), S. 235-250, hier S. 239; Hans-Jürgen Heinrichs: Einleitung. In: Ders. (Hg.): Das Mutterrecht von Johann Jakob Bachofen in der Diskussion. Überarb. Neuausg. Frankfurt a.M. 1987, S. 7-53, hier S. 12; Uwe Wesel: Der Mythos vom Matriarchat. Über Bachofens Mutterrecht und die Stellung von Frauen in frühen Gesellschaften vor der Entstehung staatlicher Herrschaft. Frankfurt a.M. 1980, S. 9f.

24 Hartmut Zinser: Mythos des Mutterrechts. Verhandlungen von drei aktuellen Theorien des Geschlechterkampfs. Münster 1996, S. 13-16; Andreas Cesena: Johann Jakob Bachofens Geschichtsdeutung (wie Anm. 23), S. 61; Brigitte Röder, Juliane Hummel u. Brigitta Kunz: Göttinnendämmerung. Das Matriarchat in archäologischer Sicht. Krummwisch 1996, S. 25. 
ferkeit und ritterlichen Gesinnung unter den Männern“. ${ }^{25}$ Die in dieser Evokation des vergangenen matriarchalen Zeitalters zu spürende Sehnsucht, die die einstige Größe dieser verschwundenen Zeit aufschimmern lässt, sorgt dafür, dass Das Mutterrecht bis heute in Kreisen rezipiert wird, die eine ganz andere Instrumentalisierung der Vorstellung vom Matriarchat im Sinne haben.

Mit ihrem Erscheinungsjahr 1861 verweist Bachofens Schrift darauf, dass in dieser Zeit die Begeisterung für Matriarchales in der Luft lag. Als Folge der Ablösung der mosaischen Chronologie erhöhte sich das Wissen um das Alter der Welt und der Menschheit, ${ }^{26}$ und so kam die Idee einer matriarchalen oder frauenzentrierten Vergangenheit auf. Seit der Mitte der 1860er Jahre verbreitete sich in der angelsächsischen Welt der kulturelle Evolutionismus, der von einer unilinearen, stufenweisen Entwicklung der gesamten Menschheit und ihrer Sozialorganisationen ausging und überzeugt war, dass in der Vergangenheit promiskuitive Sitten herrschten. Ausgangspunkt war das 1865 erschienene Buch Primitive Marriage von John Ferguson McLennan (1827-1881). Ihm ging es darum aufzuzeigen, dass die patriarchale, monogame Familie nicht die ursprüngliche soziale Einheit darstellte, sondern die Menschheit sich von der Stufe allgemeiner Promiskuität, die keinerlei Form der Ehe und Verwandtschaftssysteme kannte, zunächst zu einer Verwandtschaftsregelung über die weibliche Linie, dann zu einer Verwandtschaftsregelung über die väterliche Seite entwickelt habe. Nach McLennan stellte die erste Form der Ehe die Raubehe dar, da früher die Frauen durch weiblichen Infantizid in der Minderheit gewesen seien. ${ }^{27}$ Zahlreiche Schriften mit ähnlichem Inhalt über die Entstehung der Familie und der menschlichen Sozialform sollten im angelsächsischen Sprachraum bis zum Ende des 19. Jahrhunderts folgen.

Die Vorstellung einer graduellen Entwicklung der Menschheit geht auf die schottischen Aufklärer des späten 18. Jahrhunderts zurück, die als Väter der modernen Sozialanthropologie gelten. Sie gingen von einer Abfolge von Wildheit, Barbarei und Zivilisation im Laufe der Menschheitsgeschichte aus. ${ }^{28}$ Im Zusammenhang mit der Vorstellung einer einstigen höheren Sozialstellung der Frau ist besonders die Schrift Observations Concerning the Distinction of Ranks in Society (1771) von John Millar (1735-1801) erwähnenswert. Millar ging von einer allmählichen Degradierung der Stellung und Bedeutung der Frau in der Gesell-

25 Johann Jakob Bachofen: Das Mutterrecht. Eine Untersuchung über die Gynaikokratie der alten Welt nach ihrer religiösen und rechtlichen Natur. Hg. v. Hans-Jürgen Heinrichs. Frankfurt a.M. 1982 [1861], S. 17.

26 Donald K. Grayson: The Establishment of Human Antiquity. New York 1983, S. 5f.; Stephen Jay Gould: Time's Arrow, Time's Cycle. Cambridge 1987, hier S. 8-14.

27 John Ferguson McLennan: Primitive Marriage. Hg. v. Peter Rivière. Chicago 1970 [1865], S. 57f., 63-65; Peter Rivière: Introduction. In: Ebd., S. VII-XLVII, hier S. XX.

28 Thomas R. Trautmann: Lewis Henry Morgan and the Invention of Kinship. Berkeley 1987, S. 9; Thomas R. Trautmann: The Revolution in Ethnological Time. In: Man, New Series 27/2 (1992), S. 379-397, hier S. 381. 
schaft aus, die sich erst mit zunehmendem Grad der Zivilisation umkehre. ${ }^{29}$ Bei ihm findet sich die Interpretation, dass die einst hohe Stellung der Frau aus der Ignoranz des Zusammenhanges von Geschlechtsverkehr und Schwangerschaft resultiere und man die Kinder als zur Mutter gehörend verstand. ${ }^{30}$ Auf solchen Vorstellungen bauten die kulturellen Evolutionisten auf, wenn sie annahmen, dass in der Vergangenheit der Beitrag des Mannes zur Reproduktion nicht erkannt wurde und die monogame Ehe nicht bekannt war. Für die Entwicklung der Familie sahen sie ein Dreistufenschema - analog zu Wildheit, Barbarei und Zivilisation über allgemeine Promiskuität und Polygamie zu Monogamie. Die Mutter-KindBindung galt ihrer Auffassung nach als einzige Gewissheit und stellte aus diesem Grund den Ausgangspunkt der Familie und der Gesellschaft dar. Mit dieser Vorstellung ging ein Lob der Mutterschaft einher, die das Werden der Menschheit und ihrer Sozialstruktur ermöglichte. ${ }^{31}$ Das Lob der Mutterschaft passt in den Zeithorizont des 19. Jahrhunderts, der den Frauen kaum andere Lebensentwürfe als Ehe und Mutterschaft zubilligte.

Den Nachweis matrilinearer Verwandtschaftssysteme bei indigenen Ethnien betrachteten die kulturellen Evolutionisten als Beleg für die einstige Vormacht des weiblichen Geschlechts. Ihr Matriarchat beschränkte sich auf Matrilinearität, das heißt Systeme, die die Verwandtschaft nach der Mutter bestimmten, und eventuell Matrilokalität, also die Wohnsitznahme des Mannes bei seiner Frau. Den meisten der kulturellen Evolutionisten galt die monogame Ehe des Westens als Klimax der Entwicklung, und so ist ihren Vorstellungen ein gewisser Eurozentrismus nicht abzusprechen. ${ }^{32}$

\section{Erste Anwendungsversuche}

Die Vorstellung einer allen Menschen gemeinsamen matriarchalen Entwicklungsstufe in der Entstehungsgeschichte der Gesellschaft wurde von verschiedenen sozialen Bewegungen aufgegriffen. Die erste soziale Bewegung, die das Matriarchat in ihre Programmatik einband, war der Sozialismus. Das Matriarchat beziehungsweise die evolutionistischen Theorien galten ihm als Beweis, dass gesellschaftliche Zustände nicht gegeben, sondern konstanten Veränderungen unterworfen seien. Seine Argumentation knüpfte an die Vorstellung vom Matriarchat als

29 Simon Pembroke: The Early Human Family. In: Classical Influences on Western Thought 1650-1870. Hg. v. R. R. Bolger. Cambridge 1979, S. 275-291, hier S. 276-280.

30 John Millar: The Origin of Distinction of Ranks. Bristol 1990 [1779], S. 47.

31 Rivière: Introduction (wie Anm. 27), S. XX; Michèle Crampe-Crasnabet: Aus der Philosophie des 18. Jahrhunderts. In: Geschichte der Frauen. 5 Bde. Hg. v. Georges Duby, Michelle Perrot. Bd. 3: Frühe Neuzeit. Frankfurt a.M. 1994, S. 333-366, hier S. 344f.

32 Elizabeth Fee: The Sexual Politics of Victorian Anthropology. In: Feminist Studies 1, 3/4 (1973), S. 29-39, hier S. 34. 
egalitärer und privateigentumsfreier Gesellschaftsform an. ${ }^{33}$ Die sozialistische Perspektive auf das Matriarchat übte auf das Matriarchatskonzept einen spürbaren Einfluss aus: Das im spirituellen Feminismus verbreitete Lob des Matriarchats als egalitärer Gesellschaftsform ist wohl als direktes Erbe des Sozialismus zu betrachten.

In Frankreich erschienen im Umfeld des utopischen Frühsozialismus Schriften, die eine radikale Umgestaltung der Gesellschaft vorschlugen, mit dem Ziel sie gerechter zu gestalten. Dazu zählen die Saint-Simonisten, benannt nach ihrem Gründer Claude Henri de Rouvroy, Comte de Saint-Simon (1760-1825). Gegen Ende seines Lebens beschäftigte sich Saint-Simon zunehmend mit Religion. In seinem Le nouveau christianisme (1824) ging es ihm um eine Hinterfragung der verschiedenen Erscheinungsformen des zeitgenössischen Christentums. Allgemein lässt sich in den 1820er und 1830er Jahren in Europa ein gesteigertes Interesse am Religiösen ausmachen, das nach einer Erneuerung des Christentums strebte. ${ }^{34}$ Saint-Simon nahm nie direkt zur Frauenfrage Stellung, er ging aber von einer speziellen Form der Unterdrückung von Frauen und Arbeitern im herrschenden System aus. Seine Anhänger - die Saint-Simonisten - gingen in ihren Forderungen nach Gleichberechtigung der Frau noch weiter als er. Die Kritik der Ehe zielte hauptsächlich auf die herrschende Doppelmoral ab, die Gehorsamkeitspflicht der Frau gegenüber dem Mann und die ungleichen Besitzrechte der Eheleute. Nach Saint-Simons Tod 1825 übernahmen Barthélémy Prosper Enfantin (1796-1864) und Armand Bazard (1791-1832) die Leitung der Bewegung, 1829 wandelten sie sie in eine Kirche um. Bereits 1831 kam es zu einer Trennung von Enfantin und Bazard, weil sich Enfantin zum Priester dieser Kirche ernannte. Er und seine Ge-

33 Sehr bedeutsam und viel gelesen sind Friedrich Engels' (1820-1895) Der Ursprung der Familie, des Privateigentums und des Staates (Erstausgabe 1884) und August Bebels (18421913) Die Frau und der Sozialismus (Erstausgabe 1879, ab der dritten Auflage von 1891 übernahm Bebel Engels' Vision der matriarchalen Urzeit, siehe Edgar Weiss: Die Frau und der Sozialismus - Variationen eines Themas bei Bebel, Paulsen und Tönnies im Kontext ihrer Zeit. Kiel 1990 [Rote Reihe, Phänomenologische und hermeneutische Pädagogik, Pädagogische Anthropologie, Ethik der Erziehung 7], hier S. 1-2). In Frankreich waren die Schriften von Paul Lafargue (1840-1911, Schwiegersohn von Karl Marx) recht verbreitet, darunter La propriété. Origine et évolution (1895) oder Le droit à la paresse (1880). Direkt mit dem Thema Matriarchat beschäftigt sich sein gleichnamiger Artikel Le matriarcat, der 1886 in der Zeitschrift Nouvelle Revue erschienen ist. Ebenfalls viel gelesen in Frankreich war Benoît Malon (1841-1893); seine Hauptwerke sind La morale sociale (1886) und Le socialisme intégral (2 Bde. 1890/91). Bei Malon ist ein deutlicher Einfluss des frühsozialistischen Gedankengutes des Saint-Simonismus und des Fourierismus auszumachen, siehe Chanial Philippe: L'ère altruiste ou le socialisme selon Benoît Malon. Revue du MAUSS 31/1 (2008), S. 155-174, hier S. 157-162.

34 Elke Kleinau: Die freie Frau. Soziale Utopien des frühen 19. Jahrhunderts. Düsseldorf 1987 (Geschichtsdidaktik: Studien, Materialien 46, Reihe Frauengeschichten), S. 29-33; François Dagognet: Trois philosophies revisitées: Saint-Simon, Proudhon, Fourier. Hildesheim 1997 (Europaea Memoria. Studien und Texte zur Geschichte der europäischen Ideen, Reihe 1: Studien 1), S. 5; Christian Laval: L'ambition sociologique. Saint-Simon, Comte, Tocqueville, Marx, Durkheim, Weber. Paris 2002 (Recherches), S. 45-49. 
treuen warteten gespannt auf das Erscheinen des weiblichen Messias, denn seiner Meinung nach bedurfte die Welt zur Heilung einer Feminisierung. Die Umwandlung in eine Kirche und die Hoffnung auf den weiblichen Messias sorgten in weiten Kreisen für eine negative Einschätzung des Saint-Simonismus. ${ }^{35}$

Von der Thematik her ist die Schrift La femme est la famille (1834) von Madame E. A. Casaubon in diesem Umfeld zu verorten. Über die Verfasserin ist weiter nichts in Erfahrung zu bringen. Casaubon vertrat die Meinung, dass Kinder unabhängig vom Zivilstand der Mutter deren Familiennamen tragen sollten, somit wäre die Benachteiligung außerehelicher Kinder wie Mütter aufgehoben:

LA FEMME EST LA FAMILLE. L'enfant doit porter son nom. La certitude EST où nul doute n'existe et le fruit doit porter le nom de l'arbre qui lui donna la vie, non celui du jardinier qui y greffa le bourgeon. La fécondité est sainte. ${ }^{36}$

Casaubon singt ein Loblied auf die Mutterschaft, die im Gegensatz zur Vaterschaft immer nachweisbar sei, und sie sieht die Frauen geheiligt durch ihre Fruchtbarkeit. Ihr Engangement galt nicht nur der Familie, die Menschheit insgesamt sollte durch diese Reform, die die Bedeutung der weiblichen Fruchtbarkeit betont, friedlicher werden, Frauen in ihrer Funktion als Mütter sollten Kriege unterbinden, indem sie den Männern - oder der Menschheit insgesamt - Nächstenliebe und gegenseitigen Respekt vermittelten: „Mères, n'avez-vous pas assez souffert pour élever vos enfants? Opposez-vous donc à ces hécatombes humaines, les siècles à venir vous en béniront! Faites comprendre aux hommes qu'ils doivent s'aimer entre eux et non s'entredéchirer." ${ }^{\text {37 }}$ Dieser interessante Text geriet in Vergessenheit, obwohl er inhaltlich und von der Argumentationsweise her, die auf das edle Wesen der Frau als Mutter und als Friedensbringerin abzielt, eine gewisse Ähnlichkeit zu aktuellen Bezugnahmen auf das Matriarchat aufweist. Ganz ähnlich liegt der Fall von $L a$ politique universelle (1852) des Zeitungsmachers Emile de Girardin (1806-1881). Der Verfasser ist nicht direkt dem sozialistischen Umfeld zuzuordnen. Aber seine Schrift sieht in utopischer Weise ebenfalls eine umfassende Umgestaltung der Gesellschaft vor. Ein Kapitel befasst sich mit der Ungerechtigkeit der französischen Gesetze gegenüber außerehelich Geborenen. Die steigende Zahl illegitimer Geburten interpretierte Girardin als Folge von Industrialisierung und Urbanisierung. In bekannter Weise argumentierte er, dass mit einer Umstellung der Familienzugehörigkeit der Kinder zur Mutter das Problem der Unehelichkeit gelöst sei und die Gesellschaftsordnung auf Gewissheit basiere, zudem stärke eine solche Entwicklung die Rolle der Frau, was seiner Meinung nach zu einer Genesung der Gesamtgesellschaft führen werde:

35 Kleinau: Die freie Frau (wie Anm. 34), S. 49-51.

36 E. A. Casaubon: La femme est la famille. Paris 1834, S. 8.

37 Ebd., S. 4f., 8, 11. 
Ainsi, par la maternité, ce puissant instinct, ce noble sentiment, se régénera l'humanité. [...] La maternité est un moule déformé auquel il faut rendre sa forme si l'on veut arrêter le déclin visible des générations asservies par l'industrie. ${ }^{38}$

Casaubon wie Girardin zelebrieren die Mutterschaft als gerechte(re)s Fundament der Gesellschaft. In dieser Tendenz des Geschlechter- wie des Matriarchatsdiskurses kommt eine frühe Modernitätskritik zum Ausdruck. ${ }^{39}$ Schriften wie die beiden genannten werden heute innerhalb des Matriarchatsdiskurses nicht rezipiert. Mit ihrem Insistieren auf dem besseren Wesen der Frau, das seinen Platz in der Gesellschaft verloren habe, was den reformbedürftigen Zustand der Gesellschaft erkläre, stehen sie den gegenwärtigen Bezügen auf das Matriarchat selbst aber näher als die Schriften, auf die sich die aktuelle Argumentation mit dem Matriarchat bezieht. Gegenwärtig sind die Bezugspunkte meist Texte, die dem wissenschaftlichen Bereich zuzuordnen sind, darunter die Schriften Bachofens oder der kulturellen Evolutionisten, denen das Matriarchat als vergangene, wenn auch schillernde und manchmal melancholisch-sehnsüchtig evozierte Epoche gilt.

Auch die erste Frauenbewegung im ausgehenden 19. Jahrhundert entdeckte das Matriarchat für ihre Zwecke - vor allem in den angelsächsischen Ländern, da dort evolutionistische Sichtweisen stärker verbreitet waren. Das Matriarchat diente dieser Bewegung als Beleg dafür, dass die rechtlose Situation der Frau nicht immer die Regel gewesen sei, sondern die Frauen einst die Rechte innehatten, für die sie jetzt kämpften. Die Argumentation mit dem Matriarchat diente der ersten Frauenbewegung dazu, ihre Ansprüche auf Frauenrechte historisch zu verankern und so zu legitimieren.

Seit den 1870er Jahren kritisierten Teile der amerikanischen Frauenbewegung Kirche und Staat als Ursache der Unterdrückung der Frau. Sie suchten nach einer den Frauen gerechteren (christlichen) Religion. Als Frucht dieser Anstrengungen kann Woman, Church and State (1893) von Matilda Joslyn Gage (1826-1898) gelten. Dieses Buch, in dem sie sich gegen die von ihr als Ursache der Unterdrückung der Frau ausgemachten Institutionen Kirche und Staat wandte, gilt als ihr Hauptwerk. Ihr Kampf gegen Institutionen und Usancen, die sie als gegen Frauen gerichtet empfand, zeigte sich auch im Privaten. Nach einer in jungen Jahren geschlossenen Ehe mit Henry H. Gage, nannte sie sich zuerst Matilda E. J. Gage, doch seit den 1870er Jahren schrieb sie konsequent ihren Mädchennamen aus und nannte sich Matilda Joslyn Gage. In der Übernahme des Namens des Mannes sah sie eine die Frauen unterdrückende und sie ihrer Identität beraubende Sitte, die für die Identitätskonfusion der Frauen mitverantwortlich sei. ${ }^{40}$ Woman, Church and State beginnt mit einem Lobgesang auf das Matriarchat, das als verlorenes Paradies

38 Emile de Girardin: La politique universelle. Décrets de l'avenir. 3. Aufl. Paris 1854, S. 212.

39 Klinger: 1800 (wie Anm. 5), S. 19f.; Susanne Heine: Wiederbelebung der Göttinnen? Zur systematischen Kritik einer feministischen Theologie. 2. Aufl. Göttingen 1989, S. 29.

40 Leila Brammer: Excluded from Suffrage History. Westport 2000 (Contributions in Women's Studies 182), S. 43f. 
eingeführt wird: ,and never was justice more perfect, never civilization higher than under the Matriarchate. ${ }^{\text {"41 }}$ Bei dem Werk handelt es sich um ein frühes Stück feministischer Literatur, das zugleich eine reimaginierte Geschichte der Frauen darstellt.

Matilda Joslyn Gage ist eine wichtige, erst in den 1980er Jahren der Vergessenheit entrissene Exponentin der ersten US-amerikanischen Frauenbewegung. Seit den 1870er Jahren ging sie allerdings zunehmend auf Distanz zur Frauenbewegung, sie empfand diese nicht mehr als progressiv wegen der ihrer Meinung nach einseitigen Fokussierung auf die Erlangung des Wahlrechts. Diese zunehmende Distanzierung war mitverantwortlich dafür, dass sie als Exponentin der ersten Frauenbewegung fast vollständig vergessen wurde. Sie konzentrierte sich in der Folge mehr auf spirituelle Belange und bemühte sich in ihren Werken aufzuzeigen, was die Frauen im Laufe der Geschichte alles erreicht hatten. ${ }^{42}$ Zeitgleich begann Matilda Joslyn Gage eine Suche nach den Wurzeln der Unterdrückung der Frau, die sie im Patriarchat ausmachte: „The sacrifice of woman to man's baser passions has ever been the distinguishing characteristic of the Patriarchate. "43 Ihre Argumentation baute auf einer alle Lebensbereiche prägenden Dichotomie zwischen den beiden Gesellschaftsformen auf. Ihrer Auffassung nach richtete sich das Patriarchat explizit gegen Frauen, und sämtliche negativen Erscheinungen der Gesellschaft waren auf das Patriarchat zurückzuführen:

The theory of a male supreme God in the interests of force and authority, wars, family discord, the sacrifice of children to appease the wrath of an offended (male) deity are all due to the Patriarchate. These were practices entirely out of consonance with woman's thought and life. ${ }^{44}$

Diese Beschreibung des Patriarchats, dem die Schuld für alle Übel zugewiesen wird, weist eine Nähe zur Argumentation der zweiten Frauenbewegung auf, die oftmals das Patriarchat als Ursache sämtlicher Probleme festmachte. ${ }^{45}$ Von der zweiten Frauenbewegung wurde Joslyn Gage mit großer Begeisterung wiederentdeckt und als Vordenkerin gefeiert. Für das Revival ist besonders die Neuauflage von Woman, Church, and State von 1980 wichtig. Die Wiederentdeckung von Matilda Joslyn Gage wurde auch durch den Umstand erleichtert, dass die Familie Gage ihre Schriften und Korrespondenzen aufbewahrt hatte. ${ }^{46}$ Mary Daly (19282010) ist in Gyn/Ecology (1979) voll des Lobes für Matilda Joslyn Gage und feiert sie als „Hag“ (Hexe), was Gage in ihrem Verständnis zu einem Vorbild für selbst-

41 Matilda Joslyn Gage: Woman, Church and State. The Original Exposé of Male Collaboration against the Female Sex. Hg. v. Sally Wagner Roesch. Watertown 1980 [1893], S. 9.

42 Brammer: Excluded (wie Anm. 40), S. 1-4, 11, XIV.

43 Gage: Woman, Church and State (wie Anm. 41), S. 21.

44 Ebd.

45 Yasemine Ergas: Der Feminismus der siebziger Jahre. In: Geschichte der Frauen. 5 Bde. Hg. v. Georges Duby u. Michelle Perrot. Bd. 5: 20. Jahrhundert. Frankfurt a.M. 1995, S. 559-580, hier S. 571, 579f.

46 Brammer: Excluded (wie Anm. 40), S. 109-113. 
ständige, vom unterdrückenden Geist des Patriarchats befreite Frauen macht: „A great Hag herself, Matilda Joslyn Gage wrote with impressive erudition and passion. “47 Das Stichwort ,Hag' in diesem Lobgesang auf Gage und ihr Werk eröffnet eine weitere Ebene ihres Schaffens. Gage prägte die Ausgestaltung des Hexenbildes mit, wie es in der Wicca-Bewegung und im spirituellen Feminismus gepflegt wird. ${ }^{48}$ In einer an Jules Michelets (1798-1874) La sorcière (1862) gemahnenden Schilderung begriff sie die Hexen als Priesterinnen einer Naturreligion: „witch, primarily signified priestess, a wise superior woman who in a sylvan landscape worshipped those gods and goddesses that together governed earth and heaven. ${ }^{* 49}$ Gage war es auch, die als eine der Ersten zur Genese dieses Hexenbildes beitrug, wonach die Hexen weise Kräuterfrauen und Heilerinnen waren, die den Frauen gegen den Willen der Kirche und ihrer Repräsentanten zu schmerzfreien Geburten verhalfen. Sie war überzeugt, dass die als Hexen verfolgten Frauen über außerordentliches medizinisches Wissen verfügten: „The use of pain-destroying medicaments by women, can be traced back from five hundred to a thousand years. [...] women doctors employed anaesthetics to mitigate the pains and perils of motherhood, throwing the sufferer into a deep sleep when the child entered the world." 50 Mit solchen Ausführungen prägte sie die Formierung des in esoterischen Kreisen gegenwärtig vorherrschenden Hexenbildes und wurde von der zweiten Frauenbewegung rezipiert, die sie auch wegen ihrer Verurteilung des Patriarchats als Vorläuferin reklamierte. ${ }^{51}$

Wie sehr oft innerhalb der ersten Frauenbewegung gilt Gages Bemühung nicht so sehr den Frauen wie den Müttern, denn die Gebärfähigkeit unterscheidet die Frau fundamental und unleugbar vom Mann. Gage singt das Lied der ehemals anerkannten, mächtigen Mutterschaft in einer Gesellschaft, die der Frau als Ganzes und Heiliges huldigte:

Ancient motherhood was represented by a sphere or circle. The circle, like the mundane egg, which is but an elongated circle, contains everything in itself and is the true microcosmos. It is eternity, it is feminine, the creative force, representing spirit. ${ }^{52}$

47 Mary Daly: Gyn/Ecology. The Metaethics of Radical Feminism. Boston 1978, S. 217. Das Buch erschien 1981 auf Deutsch als Gyn/Ökologie. Eine Metaethik des radikalen Feminismus. Gage wird als „Häxe“ gelobt. Siehe dies.: Gyn/Ökologie. Eine Metaethik des radikalen Feminismus. 5. erw. Aufl. München 1991, S. 236.

48 Siehe dazu Ronald Hutton: The Triumph of the Moon. A History of Modern Pagan Witchcraft. Oxford 1999, bes. S. $205 \mathrm{ff}$.

49 Gage: Woman, Church and State (wie Anm. 41), S. 103.

50 Ebd., S. 104.

51 Felix Wiedemann: Rassenmutter und Rebellin. Hexenbilder in Romantik, völkischer Bewegung und Feminismus. Diss. Berlin. Würzburg 2007, S. 92-105; Brammer: Excluded (wie Anm. 40), S. 36.

52 Gage: Woman, Church and State (wie Anm. 41), S. 12. 
Diese Ausführungen machen deutlich, dass die Mutterschaft als identisch mit der kreativen Kraft der Frau verstanden wird. Mutterschaft steht schließlich für den Geist, der wieder als die Beseeltheit der Natur verstanden werden kann.

Elizabeth Cady Stanton (1815-1902), eine weitere wichtige Exponentin der ersten US-amerikanischen Frauenbewegung, ging davon aus, dass Frauen durch vier Beschränkungen an ihrer vollen Entwicklung gehindert wurden: Familie, Politik, Gesellschaft und Religion. Trotzdem erkannte sie an, dass die Religion den Frauen ein wichtiges Bedürfnis sei. Für diese Haltung zeugte ihre Verbindung zu Annie Besant (1847-1933), einer ehemaligen Neo-Malthusianerin und Sozialistin, die sich zur Theosophie bekehrt und nach Helena Petrovna Blavatskys (18311891) Tod die Leitung der Theosophischen Gesellschaft übernommen hatte. ${ }^{53}$ Durch Besant wurde Cady Stanton mit dem Konzept der Reinkarnation bekannt, dem sie positiv gegenüberstand. Gleichzeitig schätzte sie aber die Möglichkeit zur Veränderung der Rolle der Frau durch den Sozialismus als wahrscheinlicher ein als eine Veränderung der Stellung der Frau durch die Theosophie. Die Suche nach einer frauengemäßen Religion betrachtete sie dennoch als legitim und war federführend an einem Versuch zur Ausgestaltung einer frauengerechteren Religion beteiligt. ${ }^{54}$ Als typisches Beispiel für ein solches Unternehmen kann The Woman's Bible gelten, die Cady Stanton unter Mitarbeit anderer Frauen 1895 herausgab, mit dem Ziel, ein ausgeglichenes Bild der Frau in der Bibel zu zeichnen. Das Projekt war umstritten, die Kritik unterstellte dem Werk Blasphemie. Innerhalb der Woman's Bible wurde in der Regel das Neue Testament gnädiger beurteilt als das Alte Testament. Letzteres verstand Cady Stanton als barbarisches Zeugnis einer längst vergangenen Epoche. ${ }^{55}$

\section{IV Übernahme völkischer Ideologie}

Die zweite Frauenbewegung entdeckte die erste Frauenbewegung als Vorläuferin, und wie dieser galt ihr das Matriarchat als einst gegebenes Gesellschaftsmodell, das erneut umgesetzt werden sollte. Mit seiner Vision einer friedlichen, nachhalti-

53 Martin Green: Eine neue Ära. New Age und ,Neue Lebenszentren“ 1890-1920. In: Sinnsuche und Sonnenbad. Experimente in Kunst und Leben auf dem Monte Verità. Hg. v. Andreas Schwab u. Claudia Lafranchi. Zürich 2001, S. 205-221, hier S. 209; Ulrich Linse: Der Rebell und die ,Mutter Erde“. Asconas ,Heiliger Berg' in der Deutung des anarchistischen Bohémien Erich Mühsam. In: Monte Verità - Berg der Wahrheit. Hg. v. Harald Szeemann. Milano 1978, S. 26-37, hier S. 30.

54 Alex Owen: The Place of Enchantment. British Occultism and the Culture of the Modern. Chicago 2004, S. 24; Kathi Kern: ,Free Woman is a Divine Being, the Savior of Mankind": Stanton's Exploration of Religion and Gender. In: Elizabeth Cady Stanton. Feminist as Thinker. Hg. v. Ellen Carol Du Bois u. Richard Càndida-Smith. New York 2007, S. 93-110, hier S. 94f., 102.

55 Kern: ,Free Woman“ (wie Anm. 54), S. 93. 
gen und von Frauen geprägten Ursprungskultur Europas enthält die Argumentation mit dem Matriarchat eurozentristische Züge. In den 1980er Jahren verstärkte die Kritik von Vertreterinnen und Vertretern indigener Ethnien vor allem aus Nordamerika, die sich gegen einen vereinnahmenden Zugriff auf ihre spirituellen und kulturellen Traditionen durch eine weiße Mittelschicht verwahrten, die Tendenz esoterischer Kreise, sich auf als ,ureuropäisch“ verstandene Traditionen zu berufen. ${ }^{56}$ So fordert Zsuzsanna Budapest (*1940), eine der Gründungsfiguren der ausschließlich auf Frauen fokussierenden ,Dianic Witchcraft', in Herrin der Dunkelheit, Königin des Lichts (1980) ihre Leserschaft auf, sich auf das spirituelle Erbe der weißen Menschen zurückzubesinnen, und es neu zusammenzustellen:

Lasst uns also unser eigenes Erbe betrachten. Denn wir wissen mehr über den indianischen Schamanismus, als über unseren eigenen. Wir haben ehrbare spirituelle Wurzeln, also lasst sie uns zurückerobern! Die weißen Menschen verehrten das Leben und die Elemente der Natur; sie sagten die Zukunft voraus, indem sie die Vögel und den Wind beobachteten; sie praktizierten Runenmagie. Das Wichtigste aber, sie nutzten Gesang und Tanz als magische Werkzeuge. ${ }^{57}$

Dieses Interesse an ,eigenen“ Praktiken läuft häufig auf eine undifferenzierte Selbstbedienung bei angenommenen Traditionen hinaus, die vom vermuteten Schamanismus des Paläolithikums über spirituelle Praktiken wie das Baumkreishoroskop der Kelten und die Runenorakel der Germanen reichen.

In der ersten Frauenbewegung der deutschsprachigen Länder war der Bezug auf das Matriarchat seltener gewesen. Dafür hatte dort der Matriarchatsdiskurs Impulse aus der Lebensreform und der völkischen Bewegung des frühen 20. Jahrhunderts aufgenommen. Dies war durchaus anschlussfähig, weil in Teilen der völkischen Bewegung wiederum ein Interesse am Matriarchat vorhanden war. ${ }^{58}$ Das Matriarchat hatte dazu gedient, eine andere Gesellschaftsordnung zu entwerfen, die auf eine Unterscheidung von matriarchal und patriarchal auf der Basis von ethnischer Zugehörigkeit hinauslief. Dabei wurde für einen ursprünglich gleichberechtigten oder matriarchalen Norden optiert, der durch das östliche und südliche Patriarchat unter Druck geraten war. Auch noch innerhalb der deutschsprachigen Matriarchats-

56 Eduard Gugenberger, Roman Schweidlenka: Mutter Erde, Magie und Politik. Zwischen Faschismus und neuer Gesellschaft. Wien 1987, S. 121f.

57 Zsuzsanne E. Budapest: Herrin der Dunkelheit. Königin des Lichts. Das praktische Anleitungsbuch für die neuen Hexen. Darmstadt 2006, S. 486. Das amerikanische Original erschien ursprünglich 1980 unter dem Titel The Feminist Book of Light and Shadows und wurde als The Holy Book of Women's Mysteries neu aufgelegt.

58 Einige der wichtigen Quellen aus dem völkischen Bereich zum Thema Matriarchat: Lenore Kühn: Magna Mater. Jena 1928; Ernst Bergmann: Erkenntnisgeist und Muttergeist. Eine Soziosophie der Geschlechter. Breslau 1932; Sophie Rogge-Börner: An geweihtem Brunnen. Weimar 1928. Eine Replik auf Bergmann ist Rogge-Börners Zurück zum Mutterrecht? Leipzig 1932. In dieses Feld gehören auch die Schriften von Herman Wirth, darunter: Der Aufgang der Menschheit. Untersuchungen zur Geschichte der Religion, Symbolik und Schrift der Atlantisch-Nordischen Rasse. 2. Aufl. Jena 1934 [1928] oder Die heilige Urschrift der Menschheit. Symbolgeschichtliche Untersuchungen diesseits und jenseits des Nordatlantik. Leipzig 1932. 
literatur der zweiten Frauenbewegung kommt oft ein unreflektiertes und undifferenziertes Sich-Bedienen bei dieser ideologisch gefärbten Literatur vor. ${ }^{59}$ Auch Heide Göttner-Abendroth mischte sich in diese in den 1980er Jahren geführte Debatte ein:

Der oberflächlichste und übelste Vorwurf ist der, matriarchale Spiritualität habe etwas mit der Ideologie der Nazis und Neonazis zu tun. Diese Verdächtigung gründet sich auschließlich auf zufällige Ähnlichkeiten wie zum Beispiel die gelegentliche Beschäftigung mit germanischer Mythologie, die gelegentliche Beschreibung germanischer Symbole, der gelegentliche Rückbezug auf heimische Folklore und auf alte Feste. [...] Aber die gleichen Bilder und gleichen Themen bedeuten noch längst nicht das gleiche. ${ }^{60}$

Sie weist Vorwürfe, die Inhalte des Matriarchatsdiskurses als nationalsozialistisch kritisieren, ganz von der Hand, was einem verbreiteten Muster des Umgangs mit dieser Kritik entspricht. Die Anhängerinnen und Anhänger des Matriarchats deuten solche berechtigten Kritikpunkte als Versuche, ihre Überzeugungen zu diskreditieren und/oder als Versuche, die Frauen gegeneinander auszuspielen. ${ }^{61}$

\section{Orte des Matriarchats}

Da das Matriarchat als Tatsache der Vorgeschichte galt, zog es auch das Interesse der archäologischen Forschung auf sich. Besonders gewisse Fundkategorien und Fundorte galten als Beweise für die einstige Existenz einer matriarchalen Kultur. $\mathrm{Zu}$ den Fundgegenständen, die in diesem Sinne gedeutet wurden, zählen die paläolithischen Venusfiguren, über deren Bedeutung und Funktion inner- und außerhalb der Forschung bis heute kontrovers diskutiert wird. Neben Interpretationen als Pornographie paläolithischer Männer oder Ausdruck sexueller Nöte männlicher Jugendlicher gibt es Ansätze, die die Venusfiguren als Ahnfrauen und Göttinnen oder als erste Selbstporträts von Frauen sehen. ${ }^{62}$ Ebenso galt das Neolithikum als

59 Ilse Korotin: ,Die mythische Wirklichkeit eines Volkes‘. J. J. Bachofen, das Mutterrecht und der Nationalsozialismus. In: Der feministische ,Sündenfall'? Antisemitische Vorurteile in der Frauenbewegung. Hg. v. Charlotte Kohn-Ley. Wien 1994, S. 84-130, hier S. 111; Eva-Maria Ziege: Sophie Rogge-Börner - Wegbereiterin der Nazidiktatur und völkische Sektiererin im Abseits. In: Zwischen Karriere und Verfolgung. Handlungsräume von Frauen im nationalsozialistischen Deutschland. Hg. v. Kirsten Heinsohn, Barbara Vogel u. Ulrike Weckel. Frankfurt a.M. 1997 (Geschichte und Geschlechter 20), S. 44-77, hier S. 61-64.

60 Heide Göttner-Abendroth: Die tanzende Göttin. 2. überarb. u. erw. Aufl. München 1984 [1982], S. 285f.

61 Susannah Heschel: Konfigurationen des Patriarchats, des Judentums und des Nazismus im deutschen feministischen Denken. In: Der feministische ,Sündenfall'? (wie Anm. 59), S. 160184, hier S. 175-178; Heine: Wiederbelebung (wie Anm. 39), S. 15f.

62 Der Ansatz der Pornographie wird vertreten in: Russel Dale Guthrie: Ethological Observations from Palaeolithical Art. In: La contribution de la zoologie et de l'éthologie à l'interprétation de l'art des peuples chasseurs préhistoriques. Hg. v. Hans-Georg Bandi, Walter Huber u. MarcRoland Sauter. Fribourg 1984, S. 35-74. Ebenso vertritt er den Ansatz der paläolithischen Kunst als von Jugendlichen geschaffen in The Nature of Palaeolithic Art. Chicago 2005. Als 
matriarchal geprägt. In der Archäologie wurde bis in die Mitte des 20. Jahrhunderts von einer einheitlichen Kultur, Gesellschaft und Religion des neolithischen Europas ausgegangen, obwohl sich dessen Gestaltungsweisen in Nord- und Südeuropa deutlich unterscheiden. Im südlichen und südöstlichen Europa wurden Idole gefunden, die als Abbildungen einer Göttin interpretiert wurden, in einer Kontinuitätslinie als Nachfolgerinnen der Venusfiguren des Paläolithikums gedeutet wurden und so als Beweise für die einstige Religion einer Großen Göttin oder gar eines Matriarchats galten. In Laienkreisen sind solche Sichtweisen noch heute verbreitet. $^{63}$

Das Bild der matriarchalen Vorzeit prägten einzelne, spektakulär rezipierte Ausgrabungen, so um 1900 die Ausgrabung und umfangreiche Rekonstruktionsarbeit des Palastes von Knossos durch Sir Arthur Evans (1851-1941). Das bronzezeitliche Kreta fungierte für ihn als Paradies, geprägt durch den Kult einer Großen Göttin, die über den ewigen Zyklus von Leben und Tod wachte. Mit dieser Evokation der kretischen Vorzeit sagte Evans jedoch vermutlich mehr über seine eigenen Sehnsüchte nach einer friedlichen Welt als über die Kultur des bronzezeitlichen Kreta aus. ${ }^{64}$

Eine weitere Fundstelle, die ihrem Ausgräber als Beweis für das Matriarchat galt, war Çatal Hüyük. Der britische Archäologe James Mellaart (*1925) leitete in den 1960er Jahren die erste Ausgrabung dieser anatolischen Siedlung aus dem 8. bis 6. Jahrtausend v. Chr. Er erblickte in den Funden Hinweise auf den Kult einer Göttin des Lebens und des Todes. So begriff er diese Kultur als ,ackerbautreibende Gesellschaft mit einem Fruchtbarkeitskult, in dem eine Göttin als Hauptgottheit verehrt wurde“ ${ }^{65}$ Nach Mellaart fungierte Çatal Hüyük als Bindeglied zwischen der alten, jägerischen Lebensweise des Paläolithikums und den sesshaften, Ackerbau treibenden Kulturen des Neolithikums. In seiner Vorstellung bestand zusätzlich eine religiöse und soziale Kontinuität zur rund 5000 Jahre jüngeren bronzezeitlichen Kultur Kretas und Festlandgriechenlands. ${ }^{66}$ Die Verehrung dieser

erster schlug Leroy McDermott in den 1980er Jahren eine Interpretation der Venusfiguren als Selbstporträts vor, und zwar in seiner Dissertation: Self-Generated Information and Representation of the Human Figure During the European Upper Paleolithic. o. O. 1985.

63 Die Kritik dieser Vorstellung ist v.a. mit den Namen der beiden Archäologen Peter J. Ucko und Andrew Fleming verbunden, die im Fach in den 1960er Jahren den Abschied von dieser undifferenzierten Sichtweise einläuteten. Andrew Fleming: The Myth of the Mother-Goddess. In: World Archaeology 1/2 (1969), S. 247-261; Peter J. Ucko: The Interpretation of Prehistoric Anthropomorphic Figurines. In: The Journal of the Royal Anthropological Institute of Great Britain and Ireland 92/1 (1962), S. 38-54.

64 Siehe Kenneth Lapatin: Mysteries of the Snake Goddess. Art, Desire and the Forging of History. Boston 2002, S. 131-133; Cathy Gere: Knossos and the Prophets of Modernism. Chicago 2009, S. 22f.

65 James Mellaart: Çatal Hüyük - Stadt aus der Steinzeit. Bergisch Galdbach 1967, S. 272. Das englische Original erschien ebenfalls 1967 als Çatal Hüyük - A Neolithic Town in Anatolia.

66 Ebd., S. 272. 
Göttin drückte sich seiner Meinung nach in den Gipsreliefs und Wandverzierungen aus:

Ihre Verbindung mit dem Leben hat ihr unvermeidliches Gegenstück in ihrer Verbindung mit dem Tode. Man sieht sie einen Sohn gebären, der in zahlreichen Kultstätten in menschlicher Gestalt, als Stier oder als Widder dargestellt wird, und der der Geburt unmittelbar vorausgehende Zustand, die Schwangerschaft, kommt ebenso sehr in Statuetten wie in Gipsreliefs zum Ausdruck. Da sie wahrscheinlich auch Todesgöttin ist, wird sie von einem Raubvogel, möglicherweise einem Geier, begleitet, und ihr grimmiger Ausdruck lässt an hohes Alter denken die grimmige Alte späterer Mythologie. ${ }^{67}$

In Mellaarts Deutung galten die für Çatal Hüyük charakteristischen Verzierungen der Wände mit remodellierten Schädeln von Tieren als Symbole der Göttin und ihres Begleiters. Es handelt sich dabei hauptsächlich um Schädel von Rindern, Geiern und Füchsen. Die Rinderschädel oder Bucrania verstand er als Symbole des Männlichen.

Die Deutung der Wandverzierung in Çatal Hüyük als Abbild der Göttin und ihrer Religion fand Eingang in den spirituellen Feminismus. Die Archäologin Marija Gimbutas (1921-1994) spielte für die Argumentation mit dem Matriarchat in den 1980er und 1990er Jahren eine bedeutende Rolle, da sie durch ihren Status als Universitätsprofessorin dem Glauben an das Matriarchat einen Anstrich von Wissenschaftlichkeit zu geben vermochte. Sie bezog sich ebenfalls auf die Funde von Çatal Hüyük. Die dortigen Wandfresken nehmen in ihrer Argumentation für Religion und Kultur der Göttin einen zentralen Raum ein: Sie versteht die Bucrania als Abbilder der Göttin und schmückt diese Umdeutung weiter aus. So schreibt sie in Die Sprache der Göttin, dass der Rinderschädel den Reproduktionsorganen und ergo der Fruchtbarkeit der Göttin entspreche und sie untereinander als auswechselbar zu gelten haben:

Warum spielt der Stier unter den Symbolen des Werdens eine so herausragende Rolle? Und wie erklärt sich seine enge Assoziation mit der Göttin? Eine Antwort auf diese Frage liegt vielleicht in der auffallenden Ähnlichkeit der inneren weiblichen Geschlechtsorgane mit einem Stierkopf. ${ }^{68}$

Bei ihrer Herleitung der Überlegung, dass der Rinderschädel identisch mit den weiblichen Geschlechtsorganen sei, stützte sie sich auf die Arbeit der Kunsthistorikerin und Urgeschichtlerin Dorothy Cameron, die 1981 in Symbols of Birth and Death in the Neolithic Era ähnliche Gedankengänge verfolgte und zu dem Schluss kam, dass die patriarchale Kultur diese Ähnlichkeit bekämpfte, bis der Stier einzig als Symbol für Männlichkeit galt. ${ }^{69}$

67 Ebd., S. 234.

68 Marija Gimbutas: Die Sprache der Göttin. Das verschüttete Symbolsystem der westlichen Zivilisation. Frankfurt a.M. 1995, S. 265. Das amerikanische Original erschien 1989 u.d.T. The Language of the Goddess.

69 Dorothy O. Cameron: Symbols of Birth and Death in the Neolithic Period. London 1981, S. 4f., 43. 
Auch Überlegungen zur Heiligkeit der Gebärmutter finden sich in Schriften aus dem Spektrum des spirituellen Feminismus in großer Zahl. So gelten die im nordwestlichen Europa verbreiteten Megalithbauten des Neolithikums als Abbilder des Körpers der Göttin, worin die Menschen ihre Toten betteten, damit sie im Schoß der Mutter und Göttin zur Ruhe finden mögen. In diesem Sinne deutet auch Christa Mulack in Die Wurzeln weiblicher Macht (1996) die Gebärmutter als ersten heiligen Raum und Erfahrung, die allen Menschen gemeinsam ist:

Hier wird demnach eine Verbindung erkennbar zwischen dem Spiel heutiger Mädchen und den Empfindungen der Menschen vergangener Epochen, für die die Weiblichkeit des Raumes gar keine Frage war, zumal der Ur-Raum des Menschen - die Gebärmutter - zweifellos weiblichen Geschlechts ist. ${ }^{70}$

Aus diesem Umstand erklärt sich ihrer Meinung nach die Analogie zwischen Frau und Gefäß, da neolithische Töpfe manchmal die Form eines Frauenkörpers aufweisen. Das obige Zitat verweist darauf, dass wiederum die Vorstellung vom Überdauern gewisser matriarchaler Inhalte im Verborgenen verwendet wird. Mulack ist der Überzeugung, dass die Spiele heutiger Mädchen die Weisheit der matriarchalen Zeit enthalten. Ihrer Auffassung nach schaffen Mädchen beim Spielen am liebsten einen abgeschlossenen Raum, der symbolisch für ihren eigenen Körperraum - die Gebärmutter - steht.

Das Bild der immer wieder erwähnten Großen Göttin als Herrin über Leben und Tod sowie als Verkörperung der Natur ist seit der Mitte des 19. Jahrhunderts nachweisbar, eine Vertiefung erfuhr es aber erst ab 1900..$^{71}$ Typischerweise tritt diese Göttin in drei Gestalten auf, die für die biologischen Lebensalter der Frau stehen: Jungfrau, Mutter und Alte. Für die Ausgestaltung dieses Bildes spielte die Altertumswissenschaftlerin Jane Ellen Harrison (1850-1928) eine entscheidende Rolle. In Prolegomena to the Study of Greek Religion (1903) und weiteren Schriften legte sie dar, dass in Griechenland vor den Olympiern andere Gottheiten weiblicher, chthonischer Art verehrt wurden und entwickelte die Vorstellung, dass die Göttin in mehrfacher Form auftrat:

So far we have seen that a goddess, to the primitive Greek, took twofold form, and this twofold form, shifting and easily interchangeable is seen to resolve very simply into the two stages of a woman's life, as Maiden and Mother. ${ }^{72}$

Ihrer Interpretation nach wurden damals nur die Aspekte der Jungfrau und Mutter kultisch verehrt. ${ }^{73}$ Die Erscheinung der Göttin als Alte wurde in der ersten Hälfte des 20. Jahrhunderts noch in einen vierten Aspekt unterteilt: die Todesbringerin. Für die Vertiefung dieses Aspektes spielte der Dichter Robert Graves (1895-1985)

70 Mulack: Wurzeln (wie Anm. 11), S. 80.

71 Siehe Hutton: The Triumph of the Moon (wie Anm. 48), S. 37, 189-191.

72 Jane Ellen Harrison: Prolegomena to the Study of Greek Religion. Princeton 1991, S. 286.

73 Ebd., S. $262 f$. 
eine wichtige Rolle mit seinem Buch The White Goddess (1948), das sich mit der Bedeutung des Poeten und seiner Beziehung zur Muse der Dichtkunst, der „Weiße[n] Göttin des Todes und der Inspiration“"74 beschäftigte. Die Muse fasste er als den Zerstörung, Schmerzen und Tod bringenden Aspekt der Göttin auf, die ihre Anbeter dafür mit ihrer wertvollsten Gabe - der Poesie - belohnte.

\section{Fazit}

Der Einblick in die Argumentation mit dem Matriarchat mag aufgezeigt haben, in welch unterschiedlichen Kontexten das Konzept von einem historisch ursprünglichen Matriarchat verwendet wurde und wird. Verstand der kulturelle Evolutionismus im ausgehenden 19. Jahrhundert unter Matriarchat hauptsächlich die Feststellung der Abstammung nach der Mutter und/oder die Wohnsitznahme des Mannes bei der Frau oder ihrer Familie - zwei Erscheinungen, die heute mit den Begriffen Matrilinearität bzw. -lokalität bezeichnet werden - so ist gegenwärtig die Matriarchatsvorstellung sehr viel umfassender. Sie beinhaltet die Überzeugung von der einstigen Vormacht des weiblichen Geschlechts im sozialen, kulturellen, religiösen sowie politischen Bereich. Die Gegenüberstellung der Inhalte der Matriarchatsvorstellung zwischen Entstehungszeit und neuesten Ausprägungen zeigt, dass sich die Argumentation mit dem Matriarchat seit ihrem ersten Auftreten konstant weiterentwickelt hat, sodass die Forschung dieses Konzept heute als wandelbares Konstrukt auffasst. ${ }^{75}$ Diese Offenheit der Vorstellung vom Matriarchat, der man unterschiedliche Bedeutungen einschreiben konnte und immer noch kann, kann mit Eric Hobsbawm ,invention of tradition“ genannt werden. Damit ist das nachträgliche Konstruieren von Traditionen gemeint, die über eine postulierte Kontinuität zur Vergangenheit verfügen. ${ }^{76}$ Die Verbindung ergibt sich daraus, dass das Matriarchat historisch-konkret in der Vergangenheit vermutet wurde und noch wird. Archäologische Funde erfuhren oftmals eine entsprechende Deutung als wissenschaftliche Beweise. Auch das von Guy P. Marchal am Beispiel der Schweizer Geschichte aufgezeigte Prinzip der Gebrauchsgeschichte lässt sich auf das Matriarchat übertragen. Gebrauchsgeschichte bezeichnet einen Umgang mit Geschichte, der die Vergangenheit wie einen Gebrauchsgegenstand instrumentalisiert, dem neue Bedeutungen eingeschrieben werden können. Die vermittelten Inhalte müssen nicht richtig und historisch nachweisbar sein, sondern es reicht, wenn sie einem allgemeinen Bedürfnis oder einer Sehnsucht entsprechen.

74 Robert Graves: Die weiße Göttin. Sprache des Mythos. Berlin 1981, S. 77. Das englische Original erschien 1947 als The White Goddess.

75 Guy P. Marchal: Schweizer Gebrauchsgeschichte. Geschichtsbilder, Mythenbildung und nationale Identität. Basel 2006, S. 13f.

76 Siehe Eric Hobsbawm: Introduction. Inventing Tradition. In: The Invention of Tradition. Hg. v. dems., Terence Ranger. Cambridge 1984 (Past and Present Publications 15), S. 1-14, hier S. 1-4. 